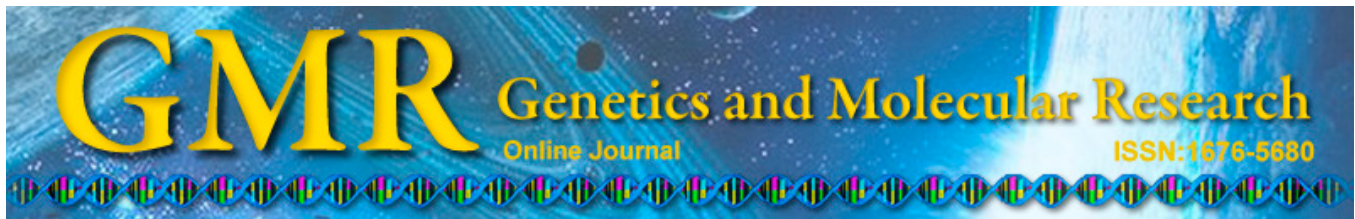

\title{
Non-parental banding patterns in recombinant inbred line population of maize with SSR markers
}

\author{
R.V. Ramekar*, K.J. Sa*, S.Y. Woo and J.K. Lee \\ Department of Applied Plant Sciences, College of Agriculture and Life Sciences, \\ Kangwon National University, Chuncheon, Korea \\ *These authors contributed equally to this study. \\ Corresponding author: J.K. Lee \\ E-mail: jukyonglee@kangwon.ac.kr
}

Genet. Mol. Res. 14 (3): 8420-8430 (2015)

Received November 17, 2014

Accepted March 18, 2015

Published July 28, 2015

DOI http://dx.doi.org/10.4238/2015.July.28.9

\begin{abstract}
We observed 3 types of non-parental banding patterns using simple-sequence repeat primers in a recombinant inbred line maize population developed from 2 inbred lines, Mo17 and KW7. We observed alleles that were not present in either of the parents, known as non-parental alleles. Although non-parental alleles are a consequence of genetic variation, they are less common in progenies derived from inbred lines. Generally, when non-parental alleles are encountered during genotyping analysis, they are either deleted from the analysis or considered to be missing data. However, before making a decision regarding how to treat non-parental alleles, it is important to understand the mechanism through which they form. There are a variety of potential reasons for the formation of non-parental bands, including recombination or mutation in the simple-sequence repeat region, residual heterozygosity in parental lines, or chromosomal aberrations resulting from rearrangements and transposons. In this article, we discuss the potential reasons behind the formation of the non-parental alleles observed in our data.
\end{abstract}

Key words: Chromosomal rearrangements; Mutation; Transposons; Non-parental alleles; Recombinants; Residual heterozygosity 


\section{INTRODUCTION}

Genetic diversity in species drives adaptation and evolution. Recombination, mutation, transposons, and chromosomal rearrangements are some of the major mechanisms that result in genetic diversity. Recombination is an important occurrence during meiosis that involves physical exchange of material between chromatids of homologous chromosome. Nonparental alleles obtained in the $\mathrm{F}_{2}$ generation are referred to as recombinants (Miglani, 2002). Mutations can create and introduce new alleles into the gene pool. Various mechanism such as substitution, insertion, deletion, and frameshifts in DNA base pairs can cause mutation and increase genetic diversity. Transposable elements and chromosome rearrangements can disrupt gene function by altering DNA sequences.

Genetic linkage maps can facilitate the development of marker-assisted selection and gene cloning (Tanksley et al., 1996; Yang et al., 2010). High-density genetic mapping using DNA probes and polymerase chain reaction-based molecular markers are essential tools for the positional cloning of genes and for providing a genetic framework for physical map construction (Tanksley et al., 1996). Recently, DNA-based molecular markers have been extensively used in the development of linkage maps, as well as in genetic research and crop breeding programs for marker-assisted selection, gene cloning, and quantitative trait locus analysis (Tanksley et al., 1996; Yang et al., 2010). Among DNA-based molecular markers, simple-sequence repeats (SSRs), which are co-dominant in nature and show a high level of polymorphism, are extensively used for constructing genetic maps and assessing genetic diversity (Akagi et al., 1997; Park et al., 2009). The Mendelian segregation ratio for co-dominant markers varies for different mapping populations, ranging from 1:2:1 for $\mathrm{F}_{2}$ progenies to $1: 1$ for recombinant inbred lines (RILs), near isogenic lines, double haploids, and backcross progenies (Xu, 2008). Particularly, in RILs, 1:1 represents equal alleles from both parents. However, in a few cases when genotyping with SSR markers, the allele represents neither parent; such alleles are referred to as non-parental alleles. Although the alleles of different genes may be linked to the same chromosome, they can be altered during meiosis. A breeding experiment carried out in Pisum sativum and Drosophila showed that even linked genes can be separated during meiosis due to exchange between chromatids of homologous chromosomes. This leads to the formation of a new or non-parental combination of genes (Jain and Sharma, 2004).

The appearance of non-parental banding patterns was suggested to be an artifact by Davis et al. (1995). After screening an $F_{2}$ mapping population with random-amplified polymorphic DNA markers, they observed a 3rd band as well as the parental bands. They suggested that this band was generated by annealing of complementary random-amplified polymorphic DNA alleles. However, Krakowsky et al. (2005), who worked with restriction fragment length polymorphism (RFLP) markers, hypothesized that the reasons underlying non-parental banding patterns in RILs may be related to contamination during inbreeding, the use of parental lines that were still segregating at some alleles, or incomplete digestion of DNA during RFLP analysis. Casa et al. (2000) suggested that non-parental fragments may be derived from residual heterozygosity, genomic rearrangements, or loss of parental variation over generations of inbreeding and mutation.

In a previous study, we constructed a framework map using SSR and single nucleotide polymorphism markers in a population of $\mathrm{F}_{7: 8}$ RILs derived from a cross between Mo17 and KW7 (Sa et al., 2012). In the previous study, we observed non-parental alleles that deviated from the parental profiles in the RIL population. In the present study, we report the non-paren- 
tal banding patterns in the maize RIL population using SSR markers and discuss the potential reasons for their formation.

\section{MATERIAL AND METHODS}

\section{Plant material and DNA isolation}

The RIL population used in this study was developed from $\mathrm{F}_{1}$ seeds, which were generated by crossing Mo17 (U.S., Corn Belt maize inbred line) and KW7 (an inbred line derived from Korean waxy corn landrace). A total of $200 \mathrm{~F}_{2}$ plants were self-pollinated and allowed to advance to the $\mathrm{F}_{7: 8}$ generation via the single-seed descent method. The final population of the $\mathrm{F}_{7: 8}$ generation consisted of 80 lines (some were lost due to sterility issues), each of which originated from different $\mathrm{F}_{2}$ individuals. DNA from the parents and the RIL mapping population were isolated from young leaves using the method described by Dellaporta et al. (1983), with minor modifications.

\section{SSR analyses}

SSR amplifications were performed in a total volume of $30 \mu \mathrm{L}$ and consisted of 20 ng genomic DNA, $1 \mathrm{X}$ polymerase chain reaction buffer, $0.3 \mu \mathrm{M}$ forward and reverse primers, $0.2 \mathrm{mM}$ dNTPs, and $1 \mathrm{U}$ Taq polymerase (Biotools, Valie de Tobalina, Madrid, Spain). The amplification procedure consisted of an initial denaturation of $94^{\circ} \mathrm{C}$ for $5 \mathrm{~min}$, followed by 2 denaturation cycles at $94^{\circ} \mathrm{C}$ for $1 \mathrm{~min}$, annealing at $65^{\circ} \mathrm{C}$ for $1 \mathrm{~min}$, and extension at $72^{\circ} \mathrm{C}$ for $2 \mathrm{~min}$. After the second cycle, the annealing temperature was decreased by $1^{\circ} \mathrm{C}$ every second cycle until the temperature reached $55^{\circ} \mathrm{C}$. The last cycle was repeated 20 times. When the cycles were complete, an extension cycle was conducted for $10 \mathrm{~min}$ at $72^{\circ} \mathrm{C}$. The SSR reaction products were added to an equal volume of stop solution $(98 \%$ deionized formamide, 2 $\mathrm{mM}$ EDTA, $0.05 \%$ bromophenol blue, $0.05 \%$ xylene cyanol) and heated at $95^{\circ} \mathrm{C}$ for $5 \mathrm{~min}$. A 3- $\mu \mathrm{L}$ aliquot of each reaction mixture was analyzed by $6 \%$ denaturing polyacrylamide gel electrophoresis and stained with silver stain.

\section{RESULTS}

\section{Screening polymorphic markers and non-parental SSR fragments}

To construct a genetic map, DNA polymorphisms between the parental lines Mo17 and KW7 were surveyed using 974 SSR primer pairs. Of these, 505 (51.8\%) pairs showed polymorphism between the parents that were used in construction of a genetic map. In our study, most SSR primers showed Mendelian segregation ratios in the RIL population and were well-distributed throughout the 10 maize chromosomes, and the positions of most of SSR markers on the maize linkage map agreed with the mapping in the MaizeGDB (http://www. maizeGDB.org). However, we observed non-parental alleles that deviated from the parental profile (Figure 1). After screening more than 505 SSR markers, 20 SSR loci (3.96\%) showed non-parental inheritance among the 80 inbred lines of the $\mathrm{F}_{7: 8}$ population (Table 1). 


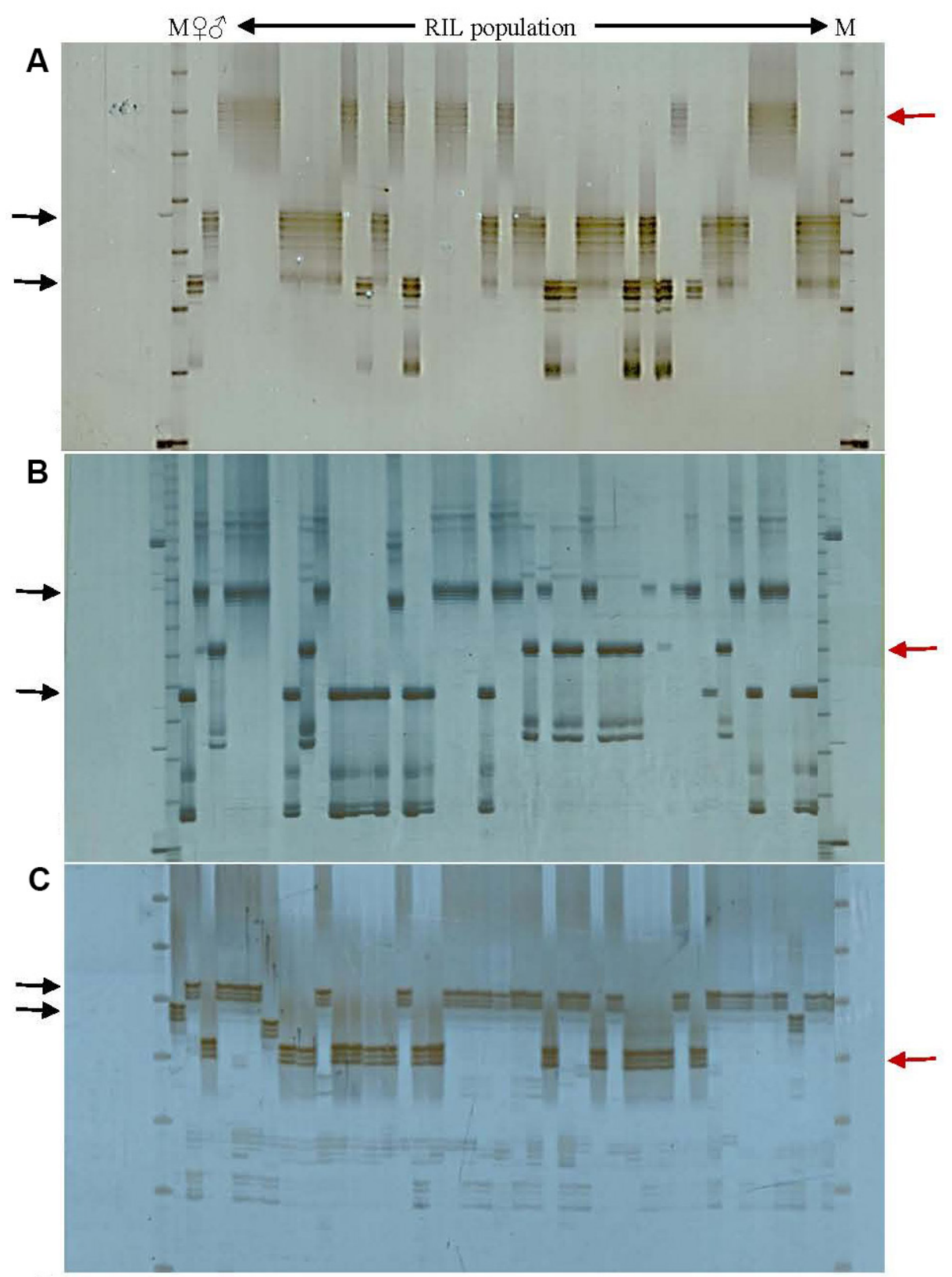

Figure 1. Examples of 3 types of non-parental alleles of SSR loci derived from the parental lines (Mo17 x KW7). A. Type A, bnlg1067: non-parental alleles showing longer fragments than parental alleles. B. Type B, umc2160: non-parental alleles showing intermediate fragments compared to parental alleles. C. Type C, umc1666: nonparental alleles showing shorter fragments compared to parental alleles. The arrows on the left side indicate alleles from the parental lines (Mo17 x KW7) of the RIL population, whereas the arrows on the right side indicate the type of non-parental alleles of the RIL population. This figure is modified from an original article (Sa et al., 2012). RIL $=$ recombinant inbred line. 
Table 1. Non-parental SSR marker information in maize RIL population.

\begin{tabular}{|c|c|c|c|c|c|}
\hline \multirow[t]{2}{*}{ No. } & \multirow[t]{2}{*}{ SSR locus } & \multirow[t]{2}{*}{ Non-parental alleles } & \multicolumn{2}{|c|}{ Number of homozygotes } & \multirow[t]{2}{*}{ Type } \\
\hline & & & Mo17 allele & KW7 allele & \\
\hline 1 & umc1282 & 42 & 0 & 38 & $\mathrm{C}$ \\
\hline 2 & bnlg1112 & 25 & 19 & 36 & $\mathrm{~B}$ \\
\hline 3 & umc2204 & 19 & 47 & 14 & B \\
\hline 4 & umc1845 & 22 & 29 & 29 & A \\
\hline 5 & umc1992 & 26 & 16 & 38 & A \\
\hline 6 & bnlg1035 & 21 & 20 & 39 & $\mathrm{C}$ \\
\hline 7 & bnlg 105 & 33 & 1 & 46 & $\mathrm{~B}$ \\
\hline 8 & umc1248 & 24 & 15 & 41 & $\mathrm{C}$ \\
\hline 9 & bnlg 1759 & 23 & 16 & 41 & $\mathrm{C}$ \\
\hline 10 & umc1066 & 25 & 21 & 34 & $\mathrm{~B}$ \\
\hline 11 & bnlg 1200 & 44 & 2 & 34 & $\mathrm{~A}$ \\
\hline 12 & umc2160 & 33 & 22 & 25 & $\mathrm{~B}$ \\
\hline 13 & bnlg 1094 & 42 & 1 & 37 & B \\
\hline 14 & umc 1549 & 41 & 1 & 38 & B \\
\hline 15 & umc 1666 & 41 & 1 & 38 & $\mathrm{C}$ \\
\hline 16 & umc 1015 & 42 & 2 & 36 & $\mathrm{~B}$ \\
\hline 17 & umc 1456 & 43 & 0 & 37 & A \\
\hline 18 & bnlg434 & 50 & 1 & 29 & B \\
\hline 19 & bnlg 1067 & 25 & 19 & 36 & A \\
\hline 20 & umc2099 & 44 & 0 & 36 & $\mathrm{C}$ \\
\hline
\end{tabular}

Type A: Non-parental alleles with longer fragments than parental alleles. Type B: Non-parental alleles with intermediate size compared to parental alleles. Type C: Non-parental alleles with shorter fragments compared to parental alleles. This table was modified from an original article (Sa et al., 2012).

\section{Non-parental banding patterns in RIL population}

We observed 3 patterns of non-parental alleles (Table 2). Type A included alleles with more base pairs when compared to the parental alleles.

Table 2. Size comparison between non-parental alleles, parental alleles, and repeat motifs of respective SSR markers.

\begin{tabular}{rllll}
\hline No. & Primer name & Repeat motifs & Type of repeat & Non-parental allele size compared to parental alleles \\
\hline 1 & umc1282 & (AT)6 & Di-nucleotide & Shorter \\
2 & bnlg1112 & AG(15) & Di-nucleotide & Intermediate \\
3 & umc2204 & (GCG)6 & Tri-nucleotide & Intermediate \\
4 & umc1845 & (AG)8 & Di-nucleotide & Longer \\
5 & umc1992 & NA & NA & Longer \\
6 & bnlg1035 & AG(13) & Di-nucleotide & Shorter \\
7 & bnlg105 & NA & NA & Intermediate \\
8 & umc1248 & (TC)12 & Di-nucleotide & Shorter \\
9 & bnlg1759 & AG(23) & Di-nucleotide & Shorter \\
10 & umc1066 & (GCCAGA)5 & Hexa-nucleotide & Intermediate \\
11 & bnlg1200 & AG(24) & Di-nucleotide & Longer \\
12 & umc2160 & (AG)10 & Di-nucleotide & Intermediate \\
13 & bnlg1094 & AG(21) & Di-nucleotide & Intermediate \\
14 & umc1549 & (GCCTCT)4 & Hexa-nucleotide & Intermediate \\
15 & umc1666 & (AG)10 & Di-nucleotide & Shorter \\
16 & umc1015 & (GA)45 & Di-nucleotide & Intermediate \\
17 & umc1456 & (AACC)5 & Tetra-nucleotide & Longer \\
18 & bnlg434 & NA & NA & Intermediate \\
19 & bnlg1067 & AG(26) & Di-nucleotide & Longer \\
20 & umc2099 & (ATGC)5 & Tetra-nucleotide & Shorter \\
\hline
\end{tabular}

$\mathrm{NA}=$ not available. 
Five SSR primers showed this pattern, including umc1845, umc1992, bnlg1200, umc1456, and bnlg1067. Type B included alleles with an intermediate number of base pairs, with an allele size between that of both parents. Nine SSR primers showed this pattern, including bnlg1112, umc2204, bnlg105, umc1066, umc2160, bnlg1094, umc1549, umc1015, and bnlg434. Type $\mathrm{C}$ included alleles that were shorter in size than the parent alleles. Six SSR primers showed this pattern, including umc1282, bnlg1035, umc1248, bnlg1759, umc1666, and umc2099.

\section{DISCUSSION}

We observed 3 patterns of non-parental alleles and categorized them as types A, B, and $\mathrm{C}$ based on their size compared with both parental alleles. Type $\mathrm{A}$ included bands that were longer than the parental alleles, type $\mathrm{C}$ were shorter than the parental alleles, and type $\mathrm{B}$ were between the sizes of the parental alleles (Figure 1). There was either an increase or decrease in the length of alleles in some RIL progeny compared with the individual lines (Mo17 or KW7).

SSR regions are considered to be hotspots for recombination (Jeffreys et al., 1998) and mutation (Vigouroux et al., 2005), which can lead to an increase or decrease in the length of alleles. In addition, residual heterozygosity in parental lines can cause deviation from the parental profile. This situation can also be caused by a chromosomal aberration resulting from rearrangement or transposon mechanisms. We will discuss each of these elements and the potential of each to play a role in the formation of the non-parental alleles observed in our data.

\section{Recombination}

Genetic recombination involves the redistribution of heritable information from parents to progeny and is instrumental in creating genetic variation. Expansion or reduction in SSR length can occur through unequal crossover (Hancock, 1996; Li et al., 2002), leading to a profile pattern for progeny samples that differs from the parental lines. For instance, bnlg1200 showed an increase in allele length in a few progeny compared to the parents, whereas the allele length of umc1282 was reduced (Table 2). It has been suggested that recombination enzymes show high affinity towards dinucleotide repeat sequences (Biet et al., 1999). Of the 20 SSR loci in our data, 12 were dinucleotide repeat motifs, mainly comprised of AT, AG, TC, and GA (Table 2). Recombination is also affected by the number of repeats. In the Escherichia coli RecA protein, binding is sequence-dependent and occurs with high affinity to GT or GC repeats (Dutreix, 1997). Thus, genetic recombination has emerged as a major source of instability for tandem repeats. Our results demonstrate that SSR regions may be affected by recombination, particularly dinucleotide repeats, which induce the formation of non-parental bands in RIL progeny.

\section{Mutation}

Mutation is a heritable change that is distinct from segregation and recombination. Mutation rates vary in maize SSRs (Vigouroux et al., 2005), but are very high compared to point mutations. Vigouroux et al. (2002) proposed that mutation may increase or decrease allele size, leading to the introduction of non-parental alleles. They sequenced the non-parental alleles and the numbers of repeat units in the putative mutants and compared them to the parental lines. At the nc009 location for 6 recombinant inbred lines in maize, they observed an $18 \mathrm{bp}$ loss or deletion of a repeat unit. They concluded that premeiotic somatic mutations in 
either the ear or the tassel cell lineage of the $\mathrm{F}_{1}$ plant gave rise to a non-parental allele, which was then inherited by recombinant inbred lines.

Slip strand mispairing errors during DNA replication may also lead to changes in the number of repeats at SSR loci. These errors are corrected by exonucleolytic proofreading and mismatch DNA repair, but many errors elude this repair mechanism and become mutations. For example, the trinucleotide $\mathrm{CTG} / \mathrm{CAG}$ or $\mathrm{CGG} / \mathrm{CCG}$ repeats can form hairpin-like structures that evade DNA repair in yeast (Hancock, 1996). If a loop occurs in the template strand and remains unrepaired, some base pairs will be deleted, leading to a strand that is shorter than the parental strand (Moore et al., 1999). If misalignment occurs in the primer strand, base pairs will be added, resulting in a strand that is longer than the parental strand (Hancock, 1996). In mice, the chance of fixation of mutations strongly depends on the selective force, which may act in favor of or against the mutated allele. If the influence of selection is ignored, a new mutation will have a 1 in 4 chance of becoming fixed in the gene pool of inbred strains (Krinke, 2000). Most SSR markers showing variations are dinucleotides (Figure 2), and because dinucleotide motifs are more prone to mutation, we should consider this as a potential causative source.

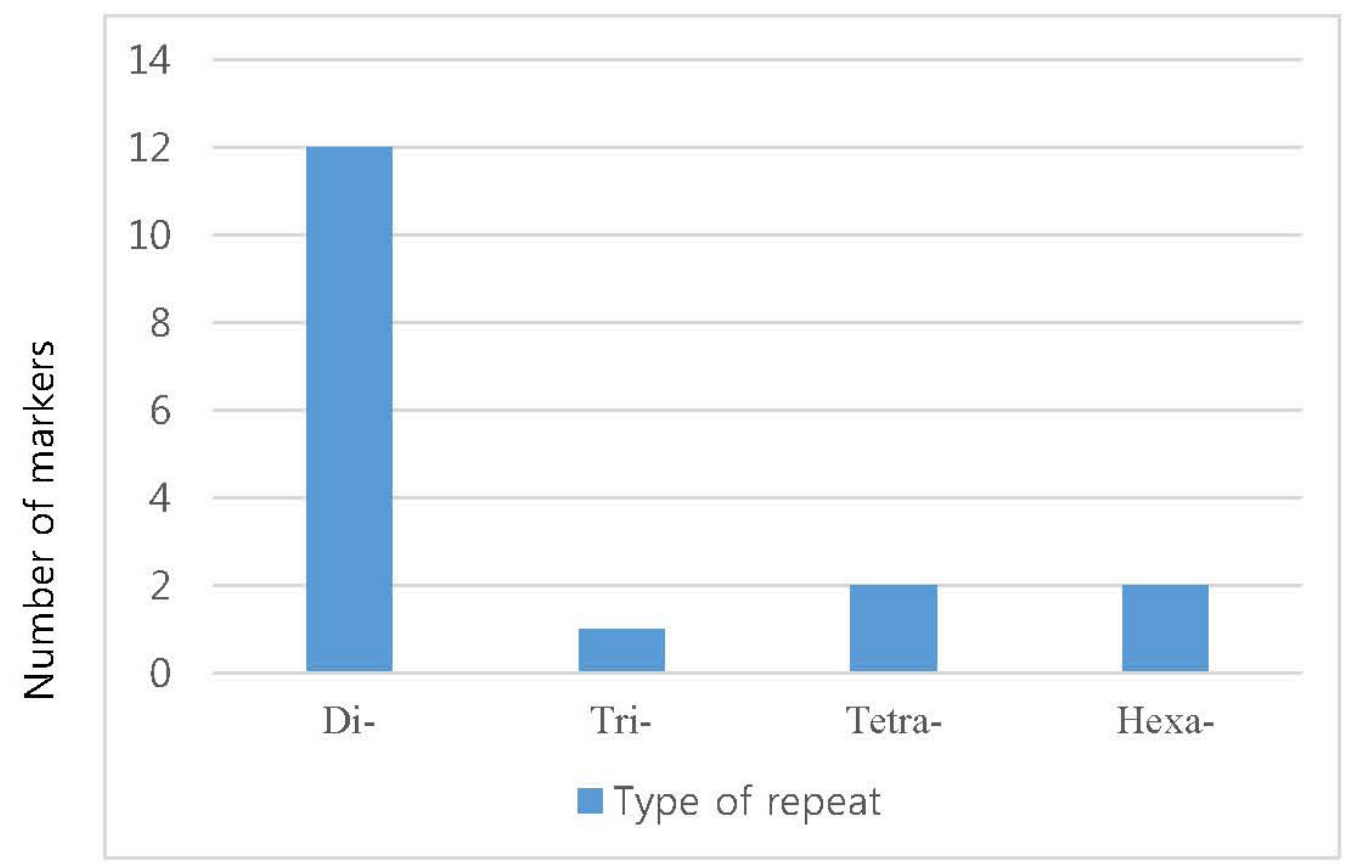

Figure 2. Graph showing distribution of SSR markers demonstrating the presence of non-parental alleles based on repeat motifs. Note that motif information for 3 markers (bnlg434, bnlg105, and umc1992) is not available.

\section{Residual heterozygosity}

Preexisting variation in parental plants may influence genetic variation and the formation of non-parental alleles (Ming, 1997; Casa et al., 2000). Thus, some variations lost in subsequent inbreeding in parental lines may be maintained in the progeny. Excess residual heterozygosity was detected by Eichten et al. (2011) in a near isogenic line population developed 
from 2 maize inbred lines (B73 and Mo17), which showed lower rates of recombination near centromeres. Inadvertent selection of plants exhibiting heterosis for self-pollination because they are healthy during the generation of RILs and near isogenic lines will result in selection for higher than expected levels of heterozygosity (Eichten et al., 2011).

A percentage of gene loci remains heterozygous despite inbreeding. In 1965, Fisher estimated the residual heterozygosity of certain inbred generations in mice. Based on his assumptions, approximately 60 generations of crosses of either brother and sister or younger parent and offspring are required for a $99 \%$ probability that a genome is completely homozygous for 20 chromosomes of $2500 \mathrm{cM}$ in length (Foster et al., 1981). No such example was found in plants, but experiments in mice suggest that although inbred lines are considered to be homogenous in a theoretical sense, advanced breeding lines or cultivars are actually heterogeneous at some level and retain residual heterozygosity. Even a moderate advantage of heterozygotes over homozygotes can inhibit the process of obtaining homozygosity. In summary, residual heterozygosity within parental inbred lines can lead to new bands in progeny that appear as non-parental bands. Thus, we cannot rule out the possibility that some non-parental alleles in our data arose from this situation.

\section{Chromosomal rearrangements}

Rearrangements in a chromosome or genome are chromosomal abnormalities caused by various mutational changes such as insertion, inversion, deletion, duplication, or translocation, which differ from traditional Watson-Crick base pair alterations $(\mathrm{Gu}$ et al., 2008). Pupilli et al. (2001) observed a non-parental banding pattern in somatic hybrid plants produced by protoplast fusion. RFLP analysis revealed various degrees of rearrangement, which are typically attributed to pre-existing somatic variability, stress induced by fusion processes, and by the union of distantly related species and mutational events induced by tissue culture. Along with chromosome loss, they also suggested gene conversion, unequal crossing over, and gene duplication as potential reasons for the appearance of new bands. Similar results were obtained by Xu and Pehu (1993) and Walters and Earle (1993), who proposed chromosomal rearrangement as the likely reason for the non-parental bands visible in somatic hybrids.

Major structural chromosome rearrangements, including deletions, duplications, translocations, and inversions, are often associated with heterochromatic regions (Raskina et al., 2008). Heterochromatins are rich in repetitive DNA sequences that are commonly subdivided into 3 distinct classes according to the size of the DNA monomer: satellite (typical monomer lengths of 140-180 to 300-360 bp), minisatellite (6-100 bp), and microsatellite (2-5 bp). The different satellite DNA sequences are organized into blocks and are present at multiple positions (Elgin, 1996). The ability of these sequences to change their copy number is thought to promote chromosomal rearrangements (Badaeva et al., 2007). For example, satellite DNA families are involved in recombination events in Drosophila (Kuhn et al., 2009) and play a central role in chromosome evolution in plants (Raskina et al., 2008). The role of several SSRs in chromosome organization has also been demonstrated in wheat and rye (Cuadrado and Schwarzacher, 1998). Rearrangements in chromosomes are random events; although we have no evidence of the marker position in the heterochromatin region, it is possible that non-parental patterns result from aberrations in chromosomes caused by rearrangement. 


\section{Transposons}

Many recombination, rearrangement, and mutation events lead to structural changes and even instability within the genome. These mutational changes and DNA rearrangements can be attributed in part to genome-inherent mobile sequences such as transposons, which are major sources of genetic variation. Transposons utilize recombination mechanisms but do not result in an exchange. Instead, a transposon moves directly from one site of the genome to another, without requiring an intermediary such as phage or plasmid DNA. This results in rearrangements that create a new sequence.

Transposable elements can directly change molecular composition. Insertions of transposable elements may create a new crossover hotspot that provokes transposable element-mediated homologous or non-homologous chromosome rearrangements (Raskina et al., 2008). Most dispersed repetitive DNA elements exhibit characteristics of transposable elements. As described above, heterochromatin regions that are involved in chromosomal rearrangements and are responsible for variation also appear to be rich in transposable elements. Non-parental banding patterns were observed by Casa et al. (2000) in maize in the screening of an RIL population to explore miniature inverted repeated transposable element as a class of molecular markers. They speculated that the causes were residual heterozygosity, in one or both parental lines, sequence variation at the flanking restriction site or the internal Hbr primer-binding site, or genomic rearrangement. Similar results were observed by Kwon et al. (2005), who used Rim 2/Hipa CACTA transposon display as a genetic marker in rice. In addition, Lee et al. (2006) observed non-parental banding patterns in an RIL population of maize derived from a cross of waxy corn (KW7) and dent corn (M017) using ISAAC transposon display markers. Among the 814 resolvable ISSAC transposon display bands, 21 bands showed non-parental inheritance. Because transposons are responsible for various chromosomal rearrangements and they participate in insertion mutagenesis (Hua Van et al., 2011; Chadha and Sharma, 2014), they may play a role in the appearance of non-parental inheritance (Lee et al., 2006).

An additional possibility for the formation of non-parental banding patterns is pollen contamination during RIL production. Liu et al. (2008) observed non-parental alleles in RILs and concluded that the non-parental allele RILs were likely caused by pollen contamination, as the panicles of RILs were not bagged at the rice-flowering stage. However, because only a few markers in our study (approximately 3.25\%) showed variation, we did not consider pollen contamination as a likely possibility. Moreover, technical problems during SSR analysis can lead to the detection of non-parental bands, and genotypic errors may mislead the data and null alleles may be scored for parental lines. However, because we crosschecked our data, this possibility is unlikely in our case.

\section{CONCLUSIONS}

Heterogeneity affects the interpretation of results during marker development and marker use, and deviations from expected profiles must be addressed and resolved. If unexpected variation is detected, the probable cause should be elucidated so that it can be determined whether to discard the result. Although a non-parental allele is a product of genetic variation and an important factor in evolution, it is less expected in heterogeneous populations with homozygous individuals derived from the continuous selfing of a hybrid of 2 inbred lines or cultivars where each individual is homozygous. A general approach to dealing with 
non-parental banding patterns in the mapping population for quantitative trait locus analysis is to either code these patterns as missing alleles or to eliminate them to avoid false-positive results. If the percentage of non-parental alleles is high in the mapping population, these lines are eliminated and not included in the analysis.

Variation at a number of loci suggests an early problem in plant breeding, particularly in the development of RILs. This may result from pollen contamination, in which case discarding the lines and excluding them from the analysis is recommended. Deviation at a single marker or a few markers when compared to parental alleles can result from different mechanisms. In our data, we observed a few SSR markers showing non-parental alleles. It may be possible that the SSR region itself was modified through recombination, mutation, or both, altering the banding patterns. The output of these mechanisms can result in either an increase or a decrease in allele size. Recombination is a major source of instability in tandem repeats. Most of the markers showing deviating results were dinucleotides, and it has been suggested that recombination enzymes show high affinity for these repeats. Similarly, dinucleotide repeats are more prone to mutations. The mechanisms by which SSRs exert their influence over gene function are as diverse as the functions of DNA. Because a certain percentage of loci remains heterozygote despite inbreeding, preexisting variation in plants may be a causative factor for genetic variation. Few references are available regarding the non-parental alleles for other mechanisms such as chromosomal rearrangements and transposons, but they may be important in the formation of non-parental bands. Sequencing of the non-parental band and comparing it to parental alleles will enable us to narrow down the potential reasons for the formation of non-parental banding patterns in our data.

\section{ACKNOWLEDGMENTS}

Research supported by a Korea Research Foundation Grant funded by the NextGeneration BioGreen "21" Program (Plant Molecular Breeding Center, \#PJ0080182014) of the Rural Development Administration, Republic of Korea, and Golden Seed Project (\#21300104-1-SBA10), Ministry of Agriculture, Food and Rural Affairs (MAFRA), Ministry of Oceans and Fisheries (MOF), Rural Development of Korea (RDA), and Korea Forest Service (KFS)

\section{REFERENCES}

Akagi H, Yokozeki Y, Inagaki A and Fujimura T (1997). Highly polymorphic microsatellites of rice consist of AT repeats, and a classification of closely related cultivars with these microsatellite loci. Theor. Appl. Genet. 94: 61-67.

Badaeva ED, Dedkova OS, Gay G, Pukhalskyi VA, et al. (2007). Chromosomal rearrangements in wheat: their types and distribution. Genome 50: 907-926.

Biet E, Sun J and Dutreix M (1999). Conserved sequence preference in DNA binding among recombination proteins: an effect of ssDNA secondary structure. Nucleic Acids Res. 27: 596-600.

Casa AM, Brouwer C, Nagel A, Wang L, et al. (2000). The MITE family Heartbreaker (Hbr): molecular markers in maize. Proc. Natl. Acad. Sci. U. S. A. 97: 10083-10089.

Chadha S and Sharma M (2014). Transposable elements as stress adaptive capacitors induce genomic instability in fungal pathogen Magnaporthe oryzae. PLoS One 9: e94415.

Cuadrado A and Schwarzacher T (1998). The chromosomal organization of simple sequence repeats in wheat and rye genomes. Chromosoma 107: 587-594.

Davis TM, Yu H, Haigis KM and Mcgowan PG (1995). Template mixing: a method of enhancing detection and interpretation of codominant RAPD markers. Theor. Appl. Genet. 91: 582-588.

Dellaporta SL, Wood J and Hicks JB (1983). A simple and rapid method for plant DNA preparation. Version II. Plant Mol. Biol. Rep. 1: 19-21. 
Dutreix M (1997). (GT)n repetitive tracts affect several stages of Rec A-promoted recombination. J. Mol. Biol. 273: 105-113. Eichten SR, Foerster JM, de Leon N, Kai Y, et al. (2011). B73-Mo17 near-isogenic lines demonstrate dispersed structural variation in maize. Plant Physiol. 156: 1679-1690.

Elgin SC (1996). Heterochromatin and gene regulation in Drosophila. Curr. Opin. Genet. Dev. 6: 193-202.

Fisher RA (1965). The Theory of Inbreeding. 2nd edn. Academic Press, Waltham.

Foster H, Small D and Fox J (1981). The mouse in Biomedical Research: History, Genetics and Wild Mice. In: Genetic Monitoring. Academic Press, Waltham.

Gu W, Zhang F and Lupski JR (2008). Mechanisms for human genomic rearrangements. Pathogenetics 1: 4.

Hancock JM (1996). Simple sequences and the expanding genome. Bioessays 18: 421-425.

Hua-Van A, Le Rouzic A, Boutin TS, Filée J, et al. (2011). The struggle for life of the genome's selfish architects. Biol. Direct 6: 19.

Jain VK and Sharma JP (2004). Comprehensive Objective Biology. In: Genes and Chromosomes. 2nd edn. Golden Bells Press, Denver.

Jeffreys AJ, Murray J and Neumann R (1998). High-resolution mapping of crossovers in human sperm defines a minisatellite-associated recombination hotspot. Mol. Cell. 2: 267-273.

Krakowsky MD, Lee M and Coors JG (2005). Quantitative trait loci for cell-wall components in recombinant inbred lines of maize (Zea mays L.) I: stalk tissue. Theor. Appl. Genet. 111: 337-346.

Krinke G (2000). The Laboratory Rat. In: Reproduction and Breeding. Academic Press, Waltham.

Kuhn GC, Teo CH, Schwarzacher T and Heslop-Harrison JS (2009). Evolutionary dynamics and sites of illegitimate recombination revealed in the interspersion and sequence junctions of two nonhomologous satellite DNAs in cactophilic Drosophila species. Heredity 102: 453-464.

Kwon SJ, Park KC, Kim JH, Lee JK, et al. (2005). Rim 2/Hipa CACTA transposon display: a new genetic marker technique in Oryza species. BMC Genet. 6: 15.

Lee JK, Park JY, Kim JH, Kwon SJ, et al. (2006). Genetic mapping of the Issac-CACTA transposon in maize. Theor. Appl. Genet. 113: 16-22.

Li YC, Korol AB, Fahima T, Beiles A, et al. (2002). Microsatellites: genomic distribution, putative functions, and mutational mechanisms: a review. Mol. Ecol. 11: 2453-2465.

Liu G, Bernhardt JL, Jia MH, Wamishe YA, et al. (2008). Molecular characterization of the recombinant inbred line population derived from a japonica-indica rice cross. Euphytica 159: 73-82.

Miglani GS (2002). Advanced Genetics. In: Recombination in Drosophila males.Alpha Science, Pangbourne, 54.

Ming R, Brewbaker JL, Pratt RC, Musket T, et al. (1997). Molecular mapping of a major gene conferring resistance to maize mosaic virus. Theor. Appl. Genet. 95: 271-275.

Moore H, Greenwell PW, Liu CP, Arnheim N, et al. (1999). Triplet repeats form secondary structures that escape DNA repair in yeast. Proc. Natl. Acad. Sci. U. S. A. 96: 1504-1509.

Park YJ, Lee JK and Kim NS (2009). Simple sequence repeat polymorphisms (SSRPs) for evaluation of molecular diversity and germplasm classification of minor crops. Molecules 14: 4546-4569.

Pupilli F, Labombarda P and Arcioni S (2001). New mitochondrial genome organization in three interspecific somatic hybrids of Medicago sativa including the parent-specific amplification of substoichiometric mitochondrial DNA units. Theor. Appl. Genet. 103: 972-978.

Raskina O, Barber JC, Nevo E and Belyayev A (2008). Repetitive DNA and chromosomal rearrangements: speciationrelated events in plant genomes. Cytogenet. Genome Res. 120: 351-357.

Sa KJ, Park JY, Park KJ and Lee JK (2012). Analysis of genetic mapping in a waxy/dent maize RIL population using SSR and SNP markers. Genes Genom. 34: 157-164.

Tanksley SD, Grandillo S, Fulton TM, Zamir D, et al. (1996). Advanced backcross QTL analysis in a cross between an elite processing line of tomato and its wild relative L. pimpinellifolium. Theor. Appl. Genet. 92: 213-224.

Vigouroux Y, Jaqueth JS, Matsuoka Y, Smith OS, et al. (2002). Rate and pattern of mutation at microsatellite loci in maize. Mol. Biol. Evol. 19: 1251-1260.

Vigouroux Y, Mitchell S, Matsuoka Y, Hamblin M, et al. (2005). An analysis of genetic diversity across the maize genome using microsatellites. Genetics 169: 1617-1630.

Walters TW and Earle ED (1993). Organellar segregation, rearrangement and recombination in protoplast fusion-derived Brassica oleracea calli. Theor. Appl. Genet. 85: 761-769.

Xu S (2008). Quantitative trait locus mapping can benefit from segregation distortion. Genetics 180: 2201-2208.

Xu YS and Pehu E (1993). RFLP analysis of asymmetric somatic hybrids between Solanum tuberosum and irradiated $S$. brevidens. Theor. Appl. Genet. 86: 754-760.

Yang X, Guo Y, Yan J, Zhang J, et al. (2010). Major and minor QTL and epistasis contribute to fatty acid compositions and oil concentration in high-oil maize. Theor. Appl. Genet. 120: 665-678. 